14.3

\title{
Диодные оптопары на основе InAsSb для сенсоров углекислого газа, работающих в режиме реального времени
}

\author{
() С.Е. Александров, Г.А. Гаврилов, А.А. Капралов, Б.А. Матвеев, М.А. Ременный, Г.Ю. Сотникова \\ Физико-технический институт им. А.Ф. Иофрфе РАН, \\ 194021 Санкт-Петербург, Россия \\ e-mail: bmat@iropt3.ioffe.ru, ioffeled@mail.ru
}

(Поступило в Редакцию 11 января 2018 г.)

\begin{abstract}
Приведены результаты исследования эффективности оптопар, выполненных на неохлаждаемых иммерсионных свето- и фотодиодах на основе твердого раствора InAsSb, обладающих низкими шумами и высоким быстродействием. Исследования показали хорошие перспективы использования предлагаемых оптопар в малогабаритных низковольтных сенсорах углекислого газа: значения пороговой чувствительности сенсора на уровне сотен ppm и погрешности измерений не хуже 5\% в широком диапазоне измерения концентраций до $10 \mathrm{v} / \mathrm{v} \%$ при высоком временном разрешении $(50 \mathrm{~ms}$ ) и малом объеме пробы (не более $50 \mathrm{ml}$ ). Достигнутые повышение быстродействия и снижение объема пробы расширяют диагностические возможности капнографии и открывают возможности использования аппаратуры в педиатрии и капнографии бокового потока, включая измерение „мгновенных“ значений концентрации $\mathrm{CO}_{2}$ в процессе дыхания.
\end{abstract}

DOI: $10.21883 / 0000000000$

\section{Введение}

Инфракрасная (ИК) спектрометрия закономерно занимает прочное место среди надежных и экспрессных методов качественного и количественного анализа состава газовых, жидких и твердых смесей веществ [1]. Для реализации на практике методов ИК спектроскопии в последние годы все более активно используется диодная (оптоэлектронная) элементная база на основе узкозонных полупроводников $\mathrm{A}^{3} \mathrm{~B}^{5}$, обеспечивающая возможность проведения оптических измерений в среднем ИК диапазоне спектра $(3-5 \mu \mathrm{m})$ [1-3]. Применительно к измерению концентрации углекислого газа одна из первых конструкций оптоэлектронных сенсоров или, другими словами оптопар, содержала светодиод (СД) с рабочей длиной волны $4.2-4.3 \mu \mathrm{m}$ на основе полупроводникового твердого раствора InAsSbP и фотосопротивление (ФС) на основе солей свинца [4]. Описанные в [4] конструкции сенсора были впоследствии неоднократно модифицированы и использованы, в частности, при разработке портативных газоанализаторов (ГА) на основе оптопар „иммерсионный СД из InAsSb-ФC на основе $\mathrm{PbSe}^{\text {“, }}$ выпускавшихся в России [5]. Иммерсионные СД были также использованы в оптико-акустических ГА, обеспечивающих низкий порог обнаружения $\mathrm{CO}_{2}$ (11 ppm при времени измерения 1 s) [6], но подверженных влиянию вибрационных помех.

Важным шагом в развитии оптических сенсоров $\mathrm{CO}_{2}$, не чувствительных к вибрациям, явилась разработка и создание неохлаждаемых иммерсионных фотодиодов (ФД) с максимумом чувствительности на длине волны $4.1-4.3 \mu \mathrm{m}$ на основе твердого раствора InAsSb, обладающих низкими шумами и высоким быстродействием [7], и оптимизация схем детектирования фототоков для низ- коомных ФД [8]. Это позволило перейти к разработке ГА $\mathrm{CO}_{2}$ на основе оптопар с минимально возможными напряжением питания и энергопотреблением при высокой точности измерений и небольшом объеме пробы [9-12]. Последнее обстоятельство крайне важно при измерениях $\mathrm{CO}_{2}$ в выдохе пациентов, особенно для новорожденных детей, имеющих малый объем легких [13]. Кроме этого, высокое быстродействие вышеуказанных сенсоров и высокая скорость газообмена в кювете малого объема являются обязательными для проведения анализа изменения концентрации $\mathrm{CO}_{2}$ в режиме реального времени (т.е. в капнографии), так как существенно расширяет диагностические возможности медико-биологических исследований и контроля [13]. Вместе с тем вопросы и обсуждение аспектов применения диодных оптопар для измерений $\mathrm{CO}_{2}$ в режиме реального времени, в частности для капнографии, практически не рассматривались в литературе.

В настоящей работе приводятся и обсуждаются свойства оптоэлектронных сенсоров на основе оптопар СД-ФД применительно к измерению концентрации $\mathrm{CO}_{2}$, включая измерение „мгновенных“ значений концентрации в процессе выдоха.

\section{1. Метрологические характеристики оптоэлектронного сенсора $\mathrm{CO}_{2}$}

Для анализа работы оптопар в недисперсионных (NDIR) оптических ГА используется предложенная ранее универсальная модель сенсора, описывающая его работу с позиций теории измерительных устройств [14]. В рамках этой модели были введены понятия аппаратной и передаточной функций [11], которые определяют все метрологические характеристики сенсора как измерите- 
ля концентрации газа. Такой подход позволяет сравнить ГА, выполненные на различной элементной базе и при различных условиях работы, определить требования к конфигурации сенсора и режимам работы излучателя и приемника для достижения предельно возможной чувствительности при заданном быстродействии.

Аппаратная функция $A(\lambda, T)$ сенсора представляет собой произведение спектральных характеристик элементов оптопары с учетом их температурных зависимостей. Аналитические выражения $A(\lambda, T)$ были получены на основе статистического анализа большого числа спектральных характеристик СД и ФД на основе InAsSb в широком температурном интервале.

При использовании оптопар со спектральными характеристиками, перекрывающими некоторую полосу $\Delta \lambda=\lambda_{2}-\lambda_{1}$, аппаратная функция сенсора содержит как информационные (поглощаемые газом), так и неинформационные (не поглощаемые газом) спектральные составляющие и позволяет установить интегральную связь между выходным сигналом сенсора и значением концентрации газа $(C, \mathrm{v} / \mathrm{v} \%)$. В качестве выходного сигнала сенсора удобно использовать безразмерную величину, пропорциональную пропусканию газовой смеси $\tau(C, L, T)$, тогда передаточная функция сенсора описывается интегральным выражением вида

$$
\tau(C, L, T)=\frac{U(C, L, T)}{U_{0}(L, T)}=\frac{\int_{\lambda 1}^{\lambda 2} A(\lambda, T) \exp [-\alpha(\lambda) L C] d \lambda}{\int_{\lambda 1}^{\lambda 2} A(\lambda, T) d \lambda},
$$

где $A(\lambda, T)=S_{\mathrm{PD}}(\lambda, T) \cdot I_{\mathrm{LED}}(\lambda, T)$ - аппаратная функция сенсора, $S_{\mathrm{PD}}(\lambda, T)$ - чувствительность ФД, $I_{\mathrm{LED}}(\lambda, T)$ - интенсивность излучения СД, $\alpha(\lambda)-$ спектральный коэффициент поглощения газа, $L-$ длина взаимодействия излучения СД с газом, $U(C, L, T)-$ выходной сигнал ФД при наличии газа, $U_{0}(L, T)-$ выходной сигнал ФД без газа.

Основной задачей модели является расчет ожидаемой передаточной функции и основных метрологических характеристик сенсора для заданной конфигурации оптопары, а также оценка влияния различных факторов (в первую очередь температуры) на стабильность его показаний. В качестве примера, на рис. 1, $a$ показано температурное смещение аппаратной функции сенсора на основе диодной оптопары с максимумом спектральной характеристики $\lambda_{\max }\left(20^{\circ} \mathrm{C}\right)=4.125 \mu \mathrm{m}$ относительно спектральной области пропускания $\mathrm{CO}_{2}$, обозначенной серым цветом в верхней части рисунка. На рис. $1, b$ представлено семейство ожидаемых передаточных функций данного сенсора, рассчитанных на основании интегрального выражения (1) для длины взаимодействия с газом $L=4 \mathrm{~cm}$ и с учетом линейчатой структуры спектра поглощения $\mathrm{CO}_{2}$. Адекватность модели для углекислого газа была ранее подтверждена экспериментальной проверкой во ВНИИМ им. Д.И. Менделеева на
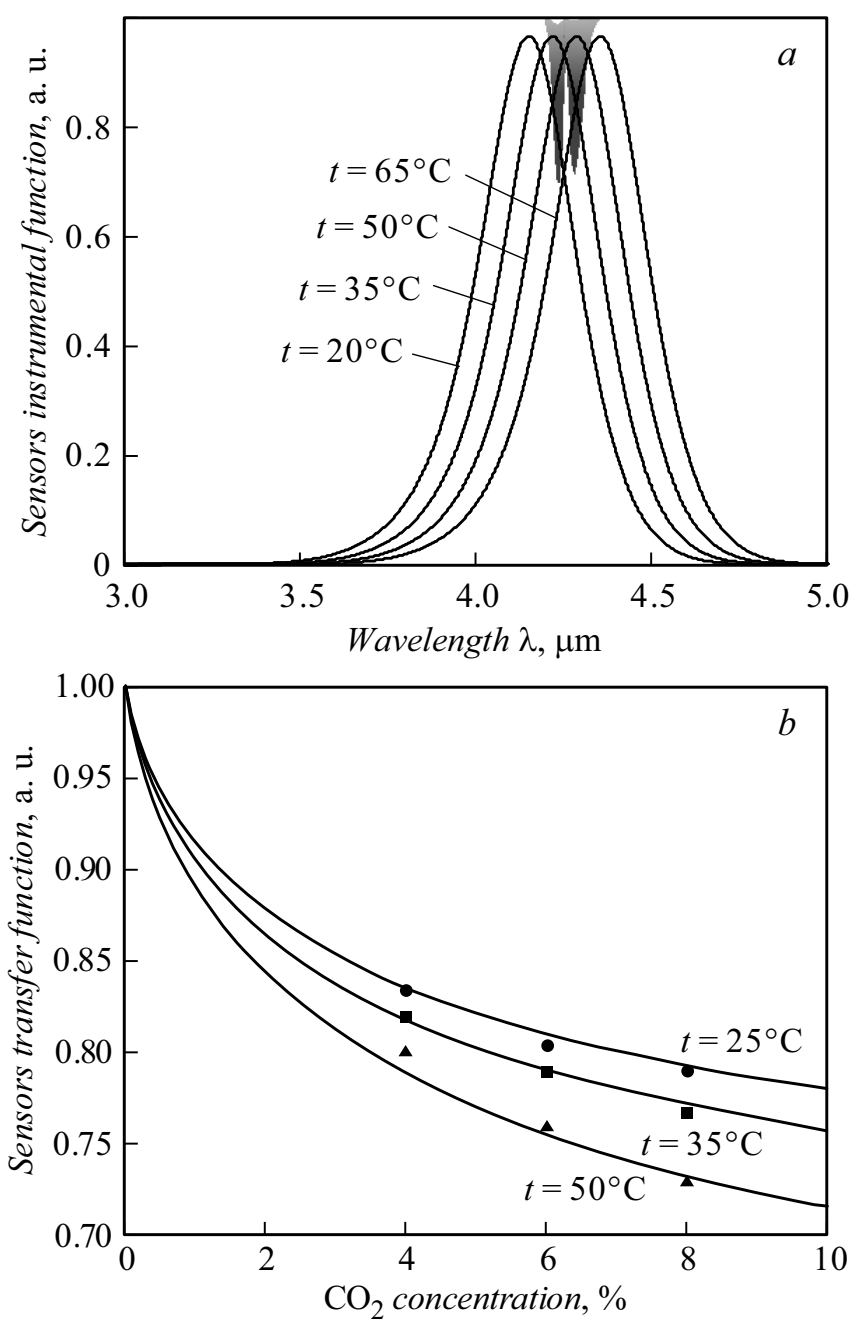

Рис. 1. Аппаратные функции сенсора на основе оптопары СД-ФД при температурах 20, 35, 50 и $65^{\circ}$ С. Для наглядности приведен спектр пропускания смеси азота и углекислого газа (a) и семейство передаточных функций сенсора для $L=4 \mathrm{~cm}$ (сплошные линии) (b). Точками представлены экспериментальные значения выходного сенсора при различных температурах в процессе его испытаний на калиброванных смесях.

калиброванных смесях $\mathrm{CO}_{2}$ и $\mathrm{N}_{2}$ в диапазоне концентраций $0.01-10 \mathrm{v} / \mathrm{v} \%[11]$.

Из рис. 1 видно, что изменение температуры приводит к перераспределению информационных и неинформационных спектральных составляющих аппаратной функции сенсора и, как следствие, к существенному изменению его передаточной функции. Таким образом, корректный расчет концентрации газа возможен либо при условии стабилизации температуры элементов оптопары, либо путем измерения температуры сенсора, например, с помощью контактного термометра и учета ее значений при дальнейших расчетах.

Решение интегрального уравнения (1) является достаточно сложной вычислительной задачей, однако для малых значений приведенных концентраций $C L, \mathrm{v} / \mathrm{v} \cdot \mathrm{cm}$ 
можно использовать приближение передаточной функции с набором эффективных значений коэффициента поглощения, $\alpha_{\mathrm{eff}}(T)$, рассчитанных для ряда рабочих температур

$$
\begin{aligned}
\tau(C, L, T) & =\frac{U(C, L, T)}{U_{0}(T)} \\
& =K(T) \exp \left[-\alpha_{\mathrm{eff}}(T) L C\right]+\tau_{0}(T),
\end{aligned}
$$

где $\tau_{0}=\tau(C=1, L, T)$ - значение выходного сигнала сенсора при температуре $T$ и 100\%-й концентрации газа, определяемое наличием неинформативных спектральных составляющих $A(\lambda, T), K(T)$ - коэффициент, определяемый при калибровке сенсора и отражающий вклад информативных спектральных составляющих $A(\lambda, T)$.

Расчеты показывают, что для сенсора углекислого газа на основе рассматриваемых диодных оптопар приближение (2) справедливо для значений $C L \leq 0.2 \mathrm{v} / \mathrm{v} \cdot \mathrm{cm}$.

Минимальное регистрируемое изменение пропускания $d \tau$ обратно пропорционально значению отношения сигнал/шум, $\Psi$, реализуемого в измерительной схеме сенсора при заданном быстродействии. Чувствительность сенсора $(d \tau / d C)$ и связанные с ней значения погрешности измерений $(d C)$ и порога обнаружения (Limit Of Detection - LOD) можно рассчитать из приближенного выражения (3)

$$
\begin{gathered}
d C=1 /(\Psi d \tau / d C) \\
=1 /\left(\Psi L \cdot \alpha_{\mathrm{eff}}(T) K(T) \exp \left(-\alpha_{\mathrm{eff}}(T) L C\right)\right), \\
\mathrm{LOD}=\frac{1}{\Psi L \alpha_{\mathrm{eff}}(T) K(T)} .
\end{gathered}
$$

Нетрудно видеть, что произведение ( $\Psi L K)$ можно рассматривать как параметр эффективности сенсора, и получение его максимально возможного значения является основной задачей при разработке газоанализаторов с предельными характеристиками по чувствительности и заданном быстродействии.

Повышение эффективности использования зондирующего ИК излучения и снижение шумов фотодетектора позволяет повысить значение $\Psi$ и уменьшить длину взаимодействия излучения с газом, сохраняя высокое значение $(\Psi L K)$. Для капнографии это приводит к значительному снижению объема пробы, забираемой у пациента, что расширяет возможности использования аппаратуры в педиатрии и капнографии бокового потока.

\section{2. Описание образцов и их характеристики}

Для создания оптопар использовались двойные гетероструктуры $p$-InAsSbP/n-InAsSb $0.08 / n$-InAs, состав и свойства которых достаточно подробно описаны в работах $[7,15,16]$. Чипы СД и ФД, имеющие круглую мезу с широким круглым анодом диаметром $\sim 0.2 \mathrm{~mm}$, и

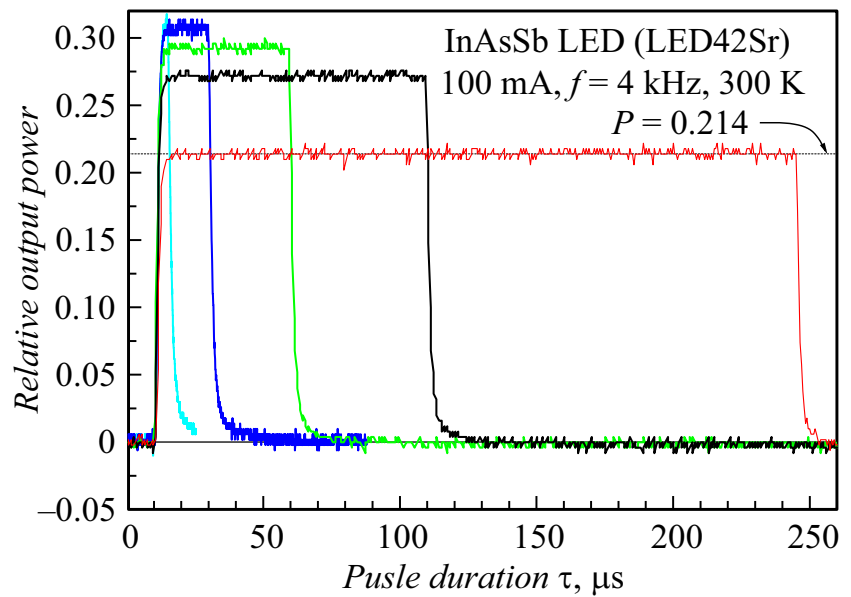

Рис. 2. Осциллограммы выходной мощности СД при $300 \mathrm{~K}$ для различных значений длительности тока накачки при его фиксированных амплитуде $(100 \mathrm{~mA})$ и частоте $(4 \mathrm{kHz})$.

катод, расположенные на эпитаксиальной поверхности подложки $n$-InAs, создавались методами стандартной фотолитографии с применением „мокрого“ химического травления. Подробную схему аналогичного чипа с описанием состава и отражательных свойств контактов, а также особенностей перераспределения в нем излучения можно найти в работе [17].

Свободная от контактов поверхность чипа с помощью халькогенидного стекла „приклеивалась“ к плоской поверхности кремниевой линзы в форме „сферы Вейерштрасса“ с просветленной поверхностью, радиусом кривизны $2.6 \mathrm{~mm}$, как было показано ранее, например в $[6,7,18]$. Диаметр линзы составлял $3.5 \mathrm{~mm}$, а диаметр открытой для излучения части - $3.2 \mathrm{~mm}$. В дальнем поле полуширина диаграммы направленности для СД составляла $\sim 10 \mathrm{deg}$ [6], при этом на расстоянии $20 \mathrm{~mm}$ от линзы формировалось увеличенное изображение активной области СД (мезы) [19]. Диаграмма направленности ФД была также узкой с полушириной $\sim 10 \mathrm{deg}$ [7]. С увеличением температуры максимумы спектров фоточувствительности ФД и излучения СД смещались в длинноволновую область спектра (рис. 1). При этом при $t=20^{\circ} \mathrm{C}$ интегральная мощность СД при токе $200 \mathrm{~mA}$ составляла $35 \mu \mathrm{W}(\mathrm{CW})$, а обнаружительная способность в максимуме спектральной характеристики ФД $-2 \cdot 10^{10} \mathrm{~cm} \cdot \mathrm{Hz}^{1 / 2} \cdot \mathrm{W}^{-1}$.

На рис. 2 приведены осциллограммы выходной мощности СД для различных значений длительности тока накачки при его фиксированных амплитуде $(100 \mathrm{~mA})$ и частоте $(4 \mathrm{kHz})$, при которой шум $1 / f$ обычно меньше теплового шума ФД. Измерения проводились в „стационарном“ режиме, т.е. после окончания переходных процессов, когда разогрев СД за счет джоулева тепла для измерений при фиксированной длительности импульса не менялся в процессе каждого выделенного импульса тока. Так, например, на рис. 2 видно хорошее совпадение мощности излучения при длительности импульса $250 \mu \mathrm{s}$ 
и постоянного уровня $P=0.214$. Отметим, что измерения проводились без принудительного охлаждения в „комфортных“ для СД условиях, когда отсутствовала его катастрофическая деградация; зона опасных для СД режимов питания, установленная эмпирически, обозначена заштрихованной областью на рис. $3, b$.

Наряду с падением мощности излучения, вызванной большой длительностью импульса, следует учитывать также и уменьшение коэффициента преобразования при увеличении тока. Это уменьшение есть фундаментальное свойство средневолновых СД, связанное с ожерекомбинацией и приводящее к сублинейности ваттамперной характеристики [16]. Последнее продемонстрировано данными на рис. $3, b$, где, в частности, показано, что более чем десятикратное увеличение амплитуды тока при длительности импульса $20 \mu$ s приводит к возрастанию импульсной оптической мощности всего лишь в 6 раз. Нелинейность ватт-амперной характеристики приводит к тому, что средняя оптическая мощность СД, или, иными словами, величина выходного сигнала оптопары также сублинейно зависит от длительности импульса тока и его амплитуды (рис. 3,a). Из данных на рис. 3, $a$ видно также, что оптимальным режимом, с точки зрения получения максимального отношения сигнал/шум при использовании СД в газовом сенсоре с частотой импульсов $f=4 \mathrm{kHz}$, является режим с небольшой скважностью и амплитудой тока $100-200 \mathrm{~mA}$.

Расходимость лучей СД накладывает ряд ограничений на тип и размеры оптической кюветы, которая ко всему прочему должна иметь минимальный объем для обеспечения возможности быстрого газообмена в капнографах бокового потока. На рис. 4 представлены экспериментальные зависимости фототока ФД от расстояния между

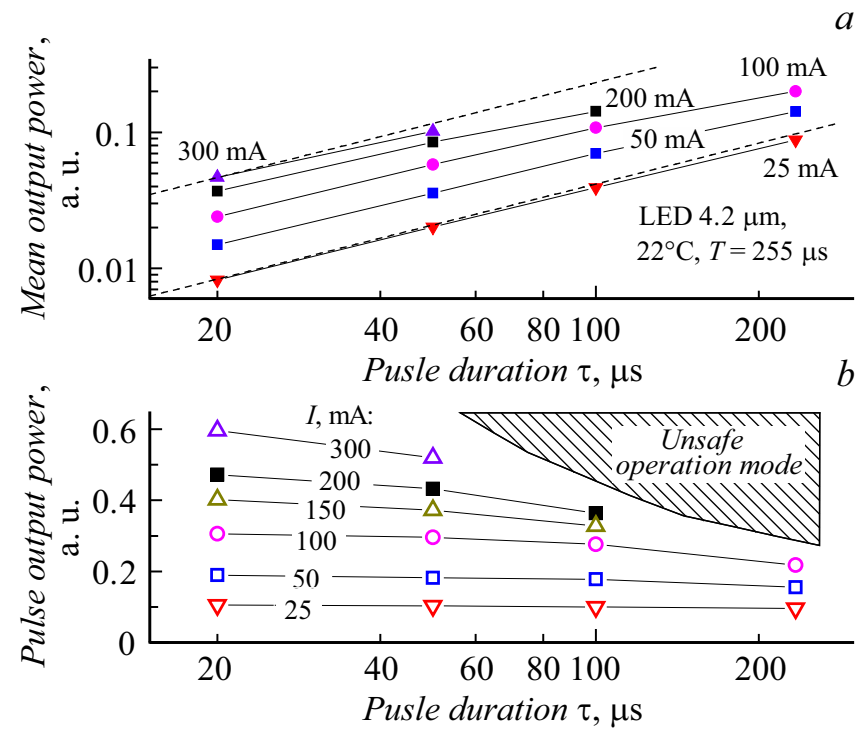

Pис. 3. Зависимости выходной средней $(a)$ и импульсной $(b)$ мощности излучения СД (LED42 Sr) от длительности импульса тока накачки. Штриховые линии - линейная зависимость вида $P_{\text {mean }} \sim \tau / T$, где $T-$ период следования импульсов.

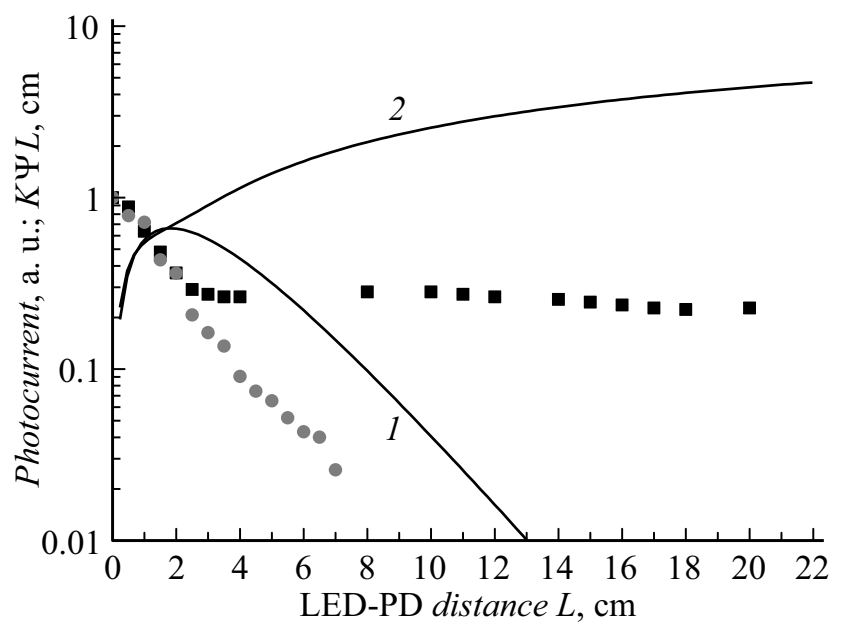

Рис. 4. Экспериментальные зависимости выходного сигнала оптопары и расчетные кривые параметра эффективности сенсора $K \Psi L$ от расстояния $L$ между СД и ФД для открытого оптического канала $(\bullet$, кривая 1$)$ и для цилиндрической алюминиевой кюветы диаметром $3.5 \mathrm{~mm}$ (ם, кривая 2).

ФД и СД при фиксированном токе через СД для случая открытого оптического канала (схема может быть использована в капнографах основного потока) и для цилиндрической алюминиевой кюветы с полированными внутренними стенками (схема для измерения бокового потока).

Полученные экспериментальные данные позволяют оценить взаимное влияние этих двух факторов на чувствительность сенсора. Сплошными линиями на рис. 4 представлены расчетные зависимости параметра эффективности сенсора $(K \Psi L)$ от расстояния $L$ между элементами оптопары (в расчетах полагалось $K=1$ ). Видно, что для свободного пространства (кривая 2) наблюдается довольно узкая область наилучшей эффективности сенсора при $L \sim 1.5-2 \mathrm{~cm}$, в то время как при использовании газовой кюветы в виде цилиндрической кюветы (кривая 1) эффективность сенсора с ростом $L$ сначала резко повышается (до $L \sim 1.5-2 \mathrm{~cm}$ ), а затем ее рост замедляется.

Измерения зависимости выходного сигнала оптопары от диаметра кюветы при $L=$ const показали, что выходной сигнал ФД остается постоянным при уменьшении ее диаметра от 3.5 до $2.5 \mathrm{~mm}$, после чего наблюдается резкое снижение сигнала. Проведенные эксперименты позволили определить оптимальный для наших компонентов размер кюветы, при котором сочетаются эффективное использование мощности зондирующего излучения СД и минимальный объем газовой пробы $(V=5 \mathrm{ml})$ при внутреннем диаметре кюветы $2.5 \mathrm{~mm}$.

\section{3. Лабораторный макет сенсора дыхания}

Для проведения экспериментальных исследований прототипа медицинского сенсора дыхания (капнографа) 
был изготовлен лабораторный макет, схема которого представлена на рис. 5.

Газовая кювета была выполнена в виде металлического цилиндра с внутренним диаметром $2.5 \mathrm{~mm}$ и $L=20 \mathrm{~mm}$ (объем $5 \mathrm{ml}$ ). Кювета снабжена юстировочными элементами, обеспечивающими согласование осей диаграмм направленности излучения СД и чувствительности ФД, которые могут иметь неконтролируемое отклонение от оси [20]. СД работал в импульсном режиме с частотой модуляции $2 \mathrm{kHz}$, при этом значение $\Psi$ было более 300 при быстродействии сенсора $50 \mathrm{~ms}$ (усреднение по 100 импульсам СД) и амплитуде импульсов тока накачки СД $200 \mathrm{~mA}$.

СД и ФД находились в тепловом контакте с газовой кюветой, температура которой измерялась с помощью контактного Pt-термометра с точностью $\pm 0.1^{\circ} \mathrm{C}$. Это позволяет производить коррекцию показаний сенсора в соответствии с семейством передаточных характеристик (2) для набора эффективных значений коэффициента поглощения газового сенсора, $\alpha_{\mathrm{eff}}(T)$. Схема детектирования сигнала ФД выполнена на элементной базе, обеспечивающей его максимальную чувствительность при минимальном температурном дрейфе в соответствии с рекомендациями, приведенными в работах $[8,21,22]$. Моделирование передаточных функций газового сенсора для $L=20 \mathrm{~mm}$ и заданных спектральных характеристик оптопары в области $4.1-4.3 \mu \mathrm{m}$ в диапазонах температур $20-50^{\circ} \mathrm{C}$ и концентраций $0-10 \mathrm{v} / \mathrm{v} \%$ позволило рассчитать ожидаемые значения $\alpha_{\mathrm{eff}}(T), \tau_{0}=\tau(C=1, L=20 \mathrm{~mm}, T=300 \mathrm{~K})$ и чувствительности сенсора к углекислому газу для $\Psi=300$. Расчеты показывают, что значения $\alpha_{\text {eff }}$ в указанном диапазоне температур меняется незначительно и составляет величину порядка $25 \mathrm{~cm}^{-1}, \tau_{0}$ лежит в диапазоне $0.5-0.75$, порог обнаружения LOD $=0.04 \mathrm{v} / \mathrm{v} \%$, относительная погрешность - не хуже $5 \%$ от измеряемой величины в диапазоне измеряемых концентраций $0-10 \mathrm{v} / \mathrm{v} \%$ при быстродействии $50 \mathrm{~ms}$ и объеме пробы $5 \mathrm{ml}$.

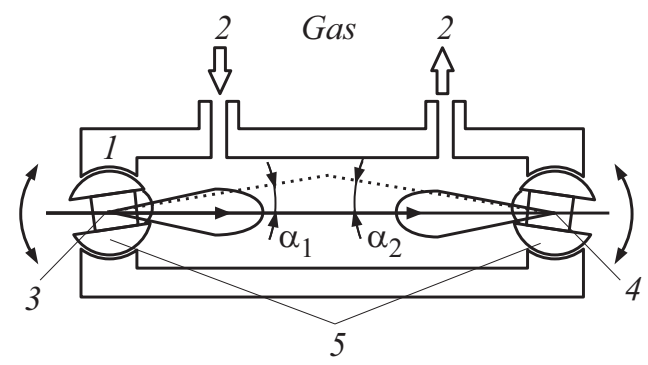

Pис. 5. Схема макета сенсора углекислого газа с использованием оптопары на основе InAsSb. 1 - цилиндрическая газовая кювета; 2 - штуцеры для прокачки газа; 3, 4 - иммерсионные СД и ФД; 5 - юстировочные элементы для согласования диаграмм направленности излучения СД и чувствительности ФД $\left(\alpha_{1}\right.$ - и $\alpha_{2}$-телесные углы диаграмм направленности СД и ФД).
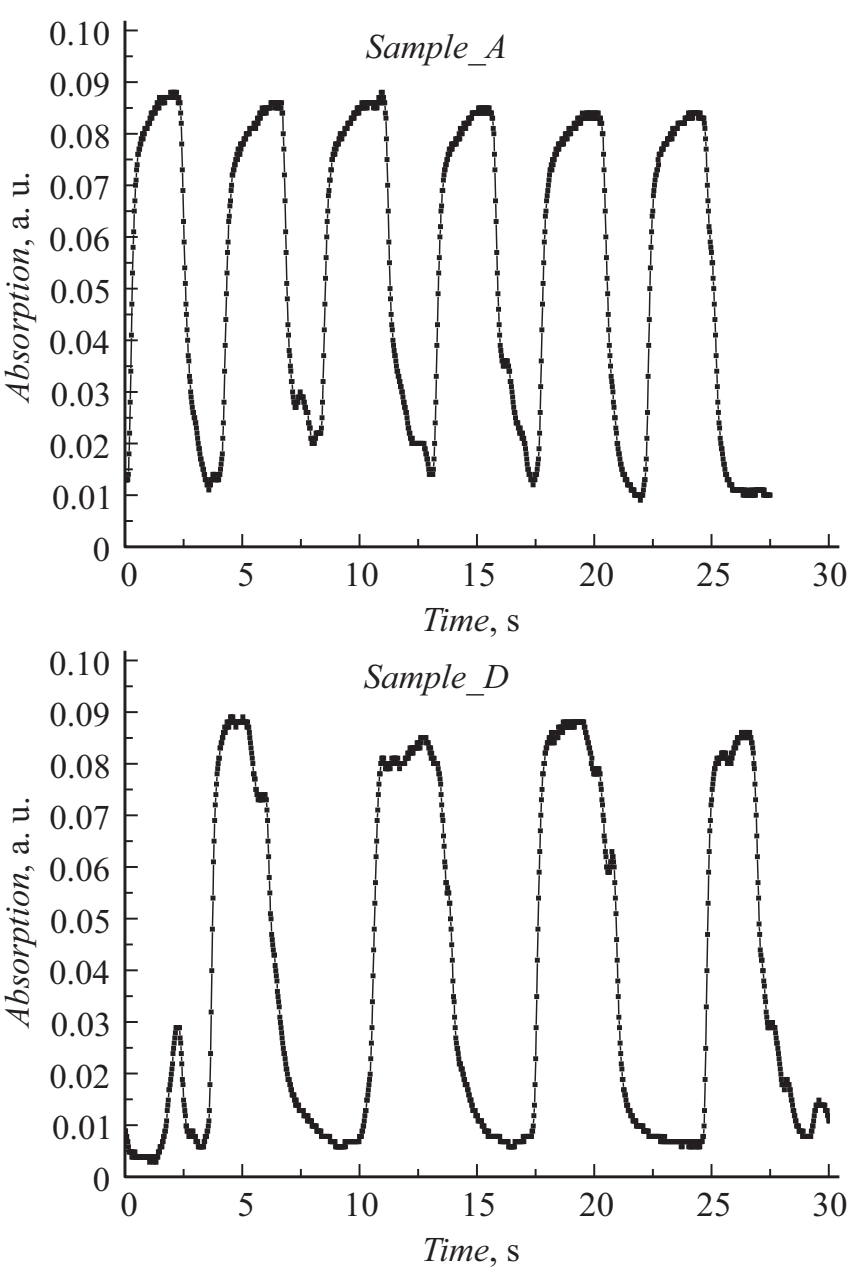

Рис. 6. Капнограммы, полученные в реальном времени с разрешением $50 \mathrm{~ms}$.

На рис. 6 приведены экспериментальные результаты мониторинга дыхания (капнограммы) двух человек, полученные в реальном времени с разрешением $50 \mathrm{~ms}$.

\section{Заключение}

Характеристики разработанного сенсора углекислого газа, содержащего диодные оптопары на основе InAsSb, соответствуют, а по ряду параметров (быстродействие и скорость газообъема в пробе) превышают лучшие образцы измерительных устройств, представленных в настоящее время на рынке медицинской аппаратуры для диагностики дыхания человека.

Конкурентные преимущества разработанных сенсоров обусловлены использованием в них отечественной элементной базы - иммерсионных диодных оптопар, обеспечивающих не только высокую чувствительность, быстродействие, помехоустойчивость, малые габариты и низкое потребление, но и возможность использования как в качестве самостоятельных приборов, так и встраивания в существующие диагностические комплексы. 


\section{Список литературы}

[1] Hodgkinson J.P., Tatam R.P. // Meas. Sci. Technol. 2013. Vol. 24. P. 012004.

[2] Zymelka D., Matveev B., Aleksandrov S., Sotnikova G., Gavrilov G., Saadaoui M. // Flex. Print. Electron. 2017. Vol. 2. P. 045006. DOI: $10.1088 / 2058-8585 / \mathrm{aa} 900 \mathrm{a}$

[3] Бинги В.Н., Степанов Е.В., Чучалин А.Г., Миляев В.А., Москаленко К.Л., Шулагин Ю.А., Янгузарова Л.Р. // Труды ин-та общей физики им. А.М. Прохорова. 2005. T. 61. C. 189.

[4] Johnston S.F. // Meas. Sci. Technol. 1992. Vol. 3. P. 191-195.

[5] Матвеев Б.А. // Фотоника. 2014. Вып. 6. С. 80-91.

[6] Kuusela T., Peura J., Matveev B.A., Remennyy M.A., Stus' N.M. // Vibrational Spectroscopy. 2009. Vol. 51. P. 289 293.

[7] Remenniy M.A., Matveev B.A., Zotova N.V., Karandashev S.A., Stus' N.M., Ilinskaya N.D. // SPIE Proc. 2007. Vol. 6585. P. 658504. DOI: $10.1117 / 12.722847$

[8] Гаврилов Г.А., Матвеев Б.А., Сотникова Г.Ю. // ПЖТФ, 2011. Т. 37. Вып. 18. С. 50-57.

[9] Avdonkin K.J., Makukha V.K. // Proceedings of the 6th Annual International Siberian Workshop on Electron Devices and Materials. 2005. P. 153-154.

[10] Yoo J., Prikhodko V., Parks J.E., Perfetto A., Geckler S., Partridge W.P. // Applied Spectroscopy. 2015. Vol. 69. P. 1047-1058. DOI: 10.1366/14-07796

[11] Sotnikova G.Y., Gavrilov G.A. Aleksandrov S.E., Kapralov A.A., Karandashev S.A., Matveev B.A., Remennyy M.A. // IEEE Sensors Journ. 2010. Vol. 10. P. 225-234. DOI: $10.1109 /$ JSEN.2009.2033259

[12] Безъязычная Т.В., Богданович М.В., Кабанов В.В., Кабанов Д.М., Лебедок Е.В., Парашук В.В., Рябцев А.Г., Рябцев Г.И., Шиак П.В., Щемелев М.А., Андреев И.А., Куницына Е.В., Шерстнев В.В., Яковлев Ю.П. // ФТП. 2015. Т. 49. Вып. 7. С. 1003-1005.

[13] Capnography, Second addition / Ed. by J.S. Gravenstein, M.B. Jaffe, N. Gravenstein, D.A. Paulus. Published by Cambridge University Press, 2011. 473 p.

[14] Электрические измерения неэлектрических величин / Л.: Энергия, 1975. 576 с.

[15] Ильинская Н.Д., Карандашев С.А., Карпухина Н.Г., Матвеев Б.А., Ременный М.А., Стусь Н.М, Усикова А.А. // ФТП. 2016. Т. 50. Вып. 5. С. 657-662.

[16] Зотова Н.В., Ильинская Н.Д., Карандашев С.А., Матвеев Б.А., Ременный М.А., Стусь Н.М. // ФТП. 2006. Т. 40. Вып. 6. С. 717-723.

[17] Закгейм А.Л., Ильинская Н.Д., Карандашев С.А., Карпухина Н.Г., Матвеев Б.А., Ременный М.А., Стусь Н.М., Усикова А.А., Черняков А.Е. // ФТП. 2017. Т. 51. Вып. 2. C. 269-275. DOI 10.21883/FTP.2017.02.44117.8380

[18] Карандашев С.А., Матвеев Б.А., Ременный М.А., Шленский А.А., Лунин Л.С., Ратушный В.И., Корюк А.В., Тараканова Н.Г. // ФТП. 2007. Т. 41. Вып. 11. С. 1389 1394.

[19] Зотова Н.В., Карандашев С.А., Матвеев Б.А., Ременный М.A., Стусь Н.М. // Оптич. журн. 2012. Т. 79. Вып. 9. C. $60-65$.

[20] Гаврилов Г.А., Сотникова Г.Ю., Александров С.Е., Усачев С.А., Капралов А.А. // Полезная модель № 75885 по заявке № 2008113124/22 от 26.03.2008.

[21] Александров С.Е., Гаврилов Г.А., Сотникова Г.Ю. // Письма в ЖТФ. 2014. Т. 40. Вып. 16. С. 58-64.

[22] Александров С.Е., Гаврилов Г.А., Сотникова Г.Ю. // Письма в ЖТФ. 2016. Т. 42. Вып. 5. С. 81-88. 